\title{
The culture and establishment of embryonic germ (EG) cell lines from Chinese mini swine
}

\author{
Hsiao Chien TSUnG ${ }^{1, *}$, Zhong Wei DU**, Rong RUI***, Xiu Lan LI ${ }^{1}$, Lin Ping BAO ${ }^{1}$, Jun WU, Shi Min \\ $\mathrm{BAO}^{2}$, ZHEN $\mathrm{YAO}^{1}$ \\ ${ }^{1}$ Laboratory of Embryonic Stem Cell, Institute of Biochemistry and Cell Biology, Shanghai Institutes for Biologcal \\ Sciences, Chinese Academy of Sciences, Shanghai 200031, China \\ ${ }^{2}$ Shanghai Laboratory Animal Center, Chinese Academy of Sciences, Shanghai 200233, China
}

\begin{abstract}
As a part of a basic research project on Xeno-transplantion, we have been engaged in the derivation of embryonic stem cell lines from Chinese mini swine. Here, we reported for the first time the establishment of two porcine EG cell lines (BPEG1 and BPEG2) from primordial germ cells of genital ridges of a 28 and a $27 \mathrm{~d}$ embryos respectively. Their pluripotent nature has been identified by colony morphology, marker characterization as well as by in vitro and in vivo differentiation. These porcine EG cells are potentially useful for further basic studies.
\end{abstract}

Key words: primordial germ cells, EG cells, porcine, Chinese mini swine.

\section{INTRODUCTION}

The clinical success of allotransplantation and the shortage of donor organs have led to the proposal for the use of animal organs as alternative therapeutic materials for human[1]. Although several major immunologic hurdles (such as hyperacute rejection) and porcine virus' s problem need to be overcome, the swine is currently considered as the most likely source of animal cells, tissues and organs for xeno-transplantation in human[2]. Particularly, mini-type swine are thought to be the most adaptive donor for organs like heart etc, from the consideration of organ size and physiology. So, a

\footnotetext{
* Correspondence: Prof. Hsiao Chien TSUNG.

Tel: 0086-21-54921367 E-mail: escg@sunm.shcnc.ac.cn

**Present address: Waisman center-University of Wisconsin T603, 1500 Highland Ave. Madison I 53705, USA. E-mail: zdu@wisc.edu

*** Who had joined and contributed to the project in the early stage while holding a postdoctoral scholarship during 1997-99, now at the College of Veterinary Medicine, Nanking Agricultural University, Jiangsu 210095, China. E-mail: rrui@njau.edu.cn Received Apr-14-2003 Revised May-16-2003 Accepted May-252003
}

lot of clinic-based studies must be performed, and among them is the estabshment of porcine embryonic stem cells and embryonic germ cells.

Mouse embryonic stem (ES) cells maintained and manipulated in vitro are able to contribute to normal embryonic and fetal development[3, 4]. In the past decade, ES cell targeting technologies have become routine procedures for mouse to introduce desired genetic change into the mouse genome[5]. Establishment of a similar system in the pig would be very useful for the production of transgenic animals devoid of undesirable antigens for xenotransplantaion. However, putative ES cells in swine have been obtained by many authors to possess some typical characteristics of pluripotent cells in short term cultures without establishing any stable cell lines[6]. So far, the only successful establishment of ES cell lines from porcine prcimplantation embryos and the demonstration of their in vivo pluripotency was the work of Chen et al[7]. In mice, primordial germ cells (PGCs) which form gametes in further fetal development are considered as an alternative source of pluripotent cell lines. Under appropriate 
in vitro conditions, these PGCs derived mouse embryonic germ cells (EG cells) give a close morphological and developmental resemblance to ES cells [8-10]. Several attempts to establish pluripotent PGCs-derived cell lines in pig have also been described and the injection of either PGC cells or shortterm cultured EG cells into recipient blastocysts and then transferred into surrogate mothers did get living chimeric offspring but without germ line transmission[11-12]. However, Mueller et al has demonstrated the presence of injected EG cells in gonad tissue of one chimeric fetus by PCR and blot methods[13]. This may indicate that in swine, like that in mice, it might be possible to realize the germ line transmission of porcine EG cells. But, it needs to be mentioned that all these authors have used common domestic pigs in their works. Nevertheless, in the absence of stable porcine ES lines, pig cloning by somatic nuclear transfer technique has made some success in recent years, e.g the production of Galknockout pig by Prather's team[14]. However, for obvious reasons, such as the abnormalities introduced by epigenetic changes during nuclear reprogramming and the low efficiency of homologous recombination in somatic cells etc., stable porcine ES or EG cell lines are still needed.

In the present study, two EG cell lines derived from Chinese mini swine have been cultured and established. The characterization of EG cells were detected by alkaline phosphatase reaction and immunohistochemical staining with monoclonal antibodies to stage specific embryonic antigens and polyclonal antibody to transcription factor Oct-4 peptides. For the validation of their pluripotency, in vitro and in vivo differentiation were also studied. The results suggest that these porcine EG cells are pluripotent and can give rise to differentiated cell types of three germ layers. The early passages of EG cells that were kept in frozen state together with STO cells can be thawed and grown without any significant loss of pluripotent properties such as growth morphology and AKP staining etc.

\section{MATERIALS AND METHODS}

\section{Isolation of primordial germ cells (PGCs)}

The swine used in this experiment was a kind of mini pig, originally introduced from Xishuangbanna by Shanghai Laboratory Animal Center, Chinese Academcy of Science in 1994. They started to inbred this strain of Chinese mini pig in the same year, reared and provided to us in 2000 .

Embryo donors were gilts of approximately 6 mon of age. Animals were slaughtered on 26-28 d of gestation, and embryos were dissected from the uteri. Genital ridge or urogenital ridge of embryos was isolated. Genitial ridges collected from each embryo were separately washed with PBS and disrupted by using fine glass needles, then incubated in $0.25 \%$ trypsin containing $0.02 \%$ EDTA or $1 \mathrm{mg} / \mathrm{ml}$ dispase (Sigma) in PBS for 5 min or for 10-15 min respectively at $37^{\circ} \mathrm{C}$. After that, PBS with $1 \%$ FCS was added and the suspension containing dissociated cells and cell fragments was centrifuged at $800 \mathrm{~g}$ for $5 \mathrm{~min}$. The sediment was resuspended in $1 \mathrm{ml}$ PEGCM (porcine EG cells culture medium): composed of DMEM containing $15 \%$ fetal bovine serum (BRL/GIBCO, special for culturing mouse ES cells), $1 \mathrm{mM}$ L-glutamine, $1 \%$ nonessential amino acid, $1 \times 10^{-4} \mathrm{M} \alpha$-mercaptoethanol, $25 \mathrm{ng} / \mathrm{ml}$ $\mathrm{rhbFGF}, 40 \mathrm{ng} / \mathrm{ml} \mathrm{rhSCF}, 10 \mathrm{ng} / \mathrm{ml} \mathrm{rhLIF}$ (all growth factors from sigma), $100 \mathrm{U} / \mathrm{ml}$ penicillin, and $0.5 \mu \mathrm{g} / \mathrm{ml}$ streptomycin.

\section{Culture of PGCs}

Feeder layer STO cells provided by Dr Piedrahita were used and passaged as described[11]. The STO cells were inactivated by the treatment with DMEM medium containing $10 \% \mathrm{FCS}$ and $10 \mu \mathrm{g} /$ $\mathrm{ml}$ mitomycin $\mathrm{C}$ (Sigma) for $2 \mathrm{~h}$ at $37^{\circ} \mathrm{C}$, and were plated at a density of $1.5 \times 10^{5}$ cells per well with coated $0.1 \%$ gelatin in a 4 -well multidish (Nunc, Roskilde, Demark).

PGC cell suspensions were separately plated on each well of 4well multidish and $0.5 \mathrm{ml}$ suspension of a pair of genetial ridges from one embryo was plated onto 2 wells. All cultures were maintained at $38^{\circ} \mathrm{C}$ in $5 \% \mathrm{CO}_{2}, 95 \%$ air with PEGCM. The medium was changed every other day after PGCs were seeded. For further subculture, the EG colonies and small fragments composed of tightly associated EG cells were passaged by trypsin or better with dispase digestion as above described, then gently dissociated by pipetting with micropipette (or mechanically by needle) and replated onto a fresh feeder layer in a 4-well multidish. EG cells of early passages were cryopreserved.

\section{Characterization of porcine EG cells}

\section{Alkaline phosphatase histochemical staining}

AKP activity of EG cells was determined during the early passages following the method described by Xu et al[15], using NBT (nitrobluetetrazolium) and BCIP (5-bromo-4-chloro-3indolyl phosphate) both from Sigma as substrate and developer. Specificity of positive staining for AKP activity was characterized by a dark violet coloration.

\section{Expression of stage-specific embryonic antigens}

Several monoclonal antibodies (McAbs) to stage specific embryonic antigens SSEA-1, SSEA-3 and SSEA-4 were purchased from the Developmental Studies Hybridoma Bank developed and maintained by the University of Iowa, Department of Biological Sciences, Iowa city, IA52242. For immunohistochemical test, the general procedure of staining was followed after Solter and Knowles for SSEA-1[16]. All above McAbs were diluted 1:10 
in PBS supplemented with 1\% BSA (Gibco) and used as first antibodies. EG cells were first transferred to gelatin-coated cover glasses with medium containing $10 \%$ (v/v) FBS. After attachment to the cover glasses for 3-10 d, EG cells or EBs grown on gelatin-coated cover glasses were fixed with $1 \%(\mathrm{w} / \mathrm{v})$ paraformadehyde at room temperature for $15 \mathrm{~min}$ and subsequently with methanol for $5 \mathrm{~min}$ at $-20^{\circ} \mathrm{C}$. After washed two times with PBS, $1 \%$ BSA was used to cover EG cells to block the nonspecific background staining for $30 \mathrm{~min}$, followed by the incubation with respective first antibodies for $1 \mathrm{~h}$ at $37^{\circ} \mathrm{C}$. Subsequently, cover glasses were incubated with rabbit anti-mouse IgG-labeled with FITC (made by our lab, 1:50 diluted in PBS) for $30 \mathrm{~min}$ at room temperature. Immunostaining was examined by fluorescence microscopy.

\section{Expression of transcription factor Oct-4}

Oct-4 is a transcription factor specifically located in nuclei of undifferentiated stem cells such as ES, EG, PGCs of genital ridge and EC (embryonal carcinoma) cells. Rabbit polyclonal antibody against Oct-4 polypeptide[17] synthesized by SBS Ltd Co. was prepared by our lab. Immunohistochemical reaction was performed by the procedure same as that of SSEA antigen staining. Oct-4 antiserum was diluted 1:20 in PBS. FITC-labeled sheep anti rabbit IgG was purchased from Dake Ltd. Co.

\section{Karyotype analysis}

Karyotype analysis was done by the method of Mueller et al [12] with modification. At passage 18-20, EG cells plated on gelatin-coated $25 \mathrm{~cm} 2$ flasks were cultured for $48 \mathrm{~h}$, and treated with $0.5 \mu \mathrm{g} / \mathrm{ml}$ colcemid (Sigma) for $5 \mathrm{~h}$ at $37^{\circ} \mathrm{C}$ in $\mathrm{CO}_{2}$. The cells were washed PBS, trypsinized with $0.25 \%$ trypsin to remove STO cells and centrifuged at $800 \mathrm{~g}$ for $5 \mathrm{~min}$. After that, hypotonization, fixation and staining of chromosomes of EG cells were followed according to the routine procedures. Karyotype evaluation and photo were taken at $1000 \times$ magnification with oil immersion.

\section{In vitro and in vivo differentiation}

EG cells are able to form embryoid bodies (EBs) when cultured in suspension for 4-5 d with PEGCM. Then EBs were collected and plated onto $0.1 \%$ gelatin-coated well $/ 4$ well muitidish or cover glasses for evaluating their developmental potential and identifying with specific marker for differentiateed cell types. DMEM containing $10 \% \mathrm{FCS}$ and $1 \times 10^{-7}-10^{-9} \mathrm{M}$ retinoic acid (RA) was used for culturing and inducing EBs differentiation for 7-10 d.

Immunohistochemisial identification of several cell types were done by methods as mentioned in the previous sections on the characterization of porcine EG cells. Neural cell typing set consisted of monoclonal antibodies against NF160 for neuron, GFAP for astrocytes and fibronectin for fibroblasts were purchased from Boehringer Mannheim Biochemica. Samples were stained for specific marker of differentiated cell types according to the manufacturer's protocol. Polyclonal antibodies to a-fetoprotein (AFP) obtained from Dako Ltd were diluted 1:10 in PBS and used for detecting AFP in endoderm cells differentiated from EBs, The staining procedure was same as that for Oct-4 staining.
Approximately $1 \times 10^{6}$ porcine EG cells (from 10 th passages) were injected subcutaneously into two axilla regions of a nude mice. After 4-5 w, tumor like outgrowths were dissected out, fixed in Bouin, sectioned and stained with HE.

\section{Scanning and transmission electron microscopic observations}

Porcine and mouse EG cells were plated separately on gelatin -coated cover glasses for scanning electron microscopy. A pellet of collected EG and STO cells was used for transmission electron microscopy. The procedure of preparations for electron microscopy was performed by usual standard techniques. Then sample observation was carried out by scanning electron-microscope HITACHI S450 and transmission electron-microscope EM OPTON 902 respectively.

\section{RESULTS AND DISCUSSION}

\section{Culture and establishment of EG cells derived from $P G C s$}

For the isolation of PGCs, embryos of Chinese mini pig were individually dissected out free from other tissues as far as possible and made into a cell suspension containing PGC cells and small cell fragments. After plated on STO cells for 5-7 d, primary putative EG colonies started to grow out, but sometime accompanied with contaminated mesonephros tissues on feeder layer (Fig 1A). After that, primary EG colonies were digested with trypsin or better with dispase and plated onto new wells with new STO cells treated by mitomycin. Numerous colonies with ES cell-like morphology could be observed after 7-10 d of sub-culture (Fig 1B), but the pig EG cell colonies were round, flatter and less translucent than mouse ES or EG colonies. Spontaneous differentiation of EG colony could occur in some passages (Fig 1C). The EG cells were passaged once a week. Thus, from 37 individual embryos dissected from 13 gilts, we have succeeded to establish two EG cell lines, designated as BPEG1 and BPEG2, derived from PGCs of a 28 d embryo and a $27 \mathrm{~d}$ embryo respectively. These two EG cell lines had been maintained for over 20 passages or 10 months by successive passaging, and still retained their characterictic growth property of early passages i.e to grow in nests similar to murine ES or EG cells and gave positive AKP staining. In one colony at $21^{\text {th }}$ passage, it was also found that their spedific markers like SSEA-3 antigen and Oct- 4 protein still reacted positively to their respective antibodies. The 
maintenance of pluripotency is also ture for frozen/ thawed EG cells of early and later passages when they were tested for growth morphology, specific markers and in vitro differentiation (data not shown).

Although the properties of EG cultures described in this paper are consistent with those of pluripotent stem cells, the cultures have a lower plating efficiency than most mouse EG and ES cell cultures. This phenomenon may be resulted from a complex set of intrinsic and extrinsic factors which include the strong adhesiveness of EG cells to resist dissociation, their lower proliferation rate as well as the influence derived from differentiated cells usually present in the same culture.

In primary culture of mouse EG cells, it is necessary to use three growth factors, including rhLIF, rhSCF and rhFGF $[8,15]$, but SCF and bFGF are no longer necessary for the passage of mouse EG cells after a cell line was already established on STO feeder cells. In the present study, the three growth factors were generally used for culturing pig PGC/
EG cells. However, we did find that in the initial culture, PGCs could also proliferate on STO feeder layer alone without the supplement of growth factors, as reported by Shim et al and Piedrahita et al[11, 12]. In the case of long-term culture experiment (successive passaging), it is better to add SCF in culture medium, unless fresh feeder layer is frequently replaced.

\section{Characterization of pig EG cells}

To characterize the undifferentiated state of EG cells, AKP staining is a routine. The early passages of EG colonies were strongly positive with AKP staining (Fig 1D). EG cells all showed positive staining when immunofluorescence reaction for SSEA1, 3 and 4 antibodies were carried out separately. However, the intensity of reaction to SSEA-1 antibodies was less strong (Fig $1 \mathrm{~F}$ ) than those to SSEA3 and SSEA-4 antibodies (Fig 1G, H). SSEA- 1 is an antigen found on mouse EC cells, ES cells and PGCs. The presence of SSEA-1 antigen in pig EG cells is similar to those findings of Takagi et al[18] and

$\triangleright$ Fig 1 (A)Primary putative EG colonies developed from genital ridges of a d 28 embryo after $7 \mathrm{~d}$ culture in vitro. Note the presence of a mesonephros tissue fragment, associated with a small EG colony. Phase contrast $\times 200$

(B) Many EG colonies at $3^{\text {rd }}$ passage on STO feeder layer. Phase contrast $\times 200$

(C) Spontaneous differentiation of EG colonies at $5^{\text {th }}$ passage into fibroblast like cells (arrows indicated). Phase contrast $\times 200$

(D) Positive AKP staining of EG colonies at $3^{\text {rd }}$ passage. $\times 200$

(E) Showing AKP positive staining of undifferentiated EG cells in the center of an embryoid body derived from a EG colony at $10^{\text {th }}$ passage with surrounding negatively stained differentiated cells. $\times 200$

(F) Immunofluorescent positive staining of EG colony at $5^{\text {th }}$ passage reacted with monoclonal antibodies against SSEA1. $\times 480$

(G) Ditto, an EG colony at $10^{\text {th }}$ passage reacted with McAb against SSEA-3. $\times 200$

(H) Ditto, an EG colony at $10^{\text {th }}$ passage reacted with McAb against SSEA-4. $\times 200$

( I ) Positive reaction of Oct-4 protein staining (Ia) of an EG cell colony at $5^{\text {th }}$ passage, (Ib) its corresponding control. Phase contrast $\times 480$

(J) Scanning electron micrographs showing that the size of pig EG cells was similar to that of mouse EG cells at the same magmification. (Ja) pig EG cells (Jb) mouse EG cells. $\times 1500$

(K) A transmission electron micrograph of a pig EG cells. $\times 9000$

(L) EBs derived from pig EG cells after attached to cover-glass for 5 d differentiated into epithelial-like cells (arrow) and fibroblast-like cells(arrowhead). Phase contrast $\times 200$

The following 4 figures (Fig 1M-1P) represented in vitro differentiation of EBs derived from EG colonies at 12-14 passages treated with $1 \times 10^{-7}-10^{-9} \mathrm{~m} M /$ RA for $5 \mathrm{~d}$, reattached to dishes for another $5-10 \mathrm{~d}$ and stained with different specific antibodies.

(M) Showing positive immuofluorescent reaction of EB and its spreading endoderm cells with anti-mouse a-fetoprotein antibodies. $\times 200$

(N) Showing positive immuochemical staining of EB derived fibroblast cells, with anti-fibronectin antibodies. $\times 200$

(O) Showing positive immuofluorescent reaction of neuron-like cells with anti-NF160 antibodies. $\times 200$

(P) Showing positive immuofluorescent reaction of astrocytes like cells with anti-GFAP antibodies. $\times 200$

(Q) Histological sections of an outgrowth obtained by the injection of $1 \times 10^{6}$ porcine BPEG1 cells into a nude mouse for $5 \mathrm{w}$, fixed and stained with HE. (Qa) Gland like structures (arrow) and neural tube like structure (arrowhead), (Qb) muscles (arrow), (Qc) neural tube like structures (arrow) and lipocytes (arrowhead). ×100 

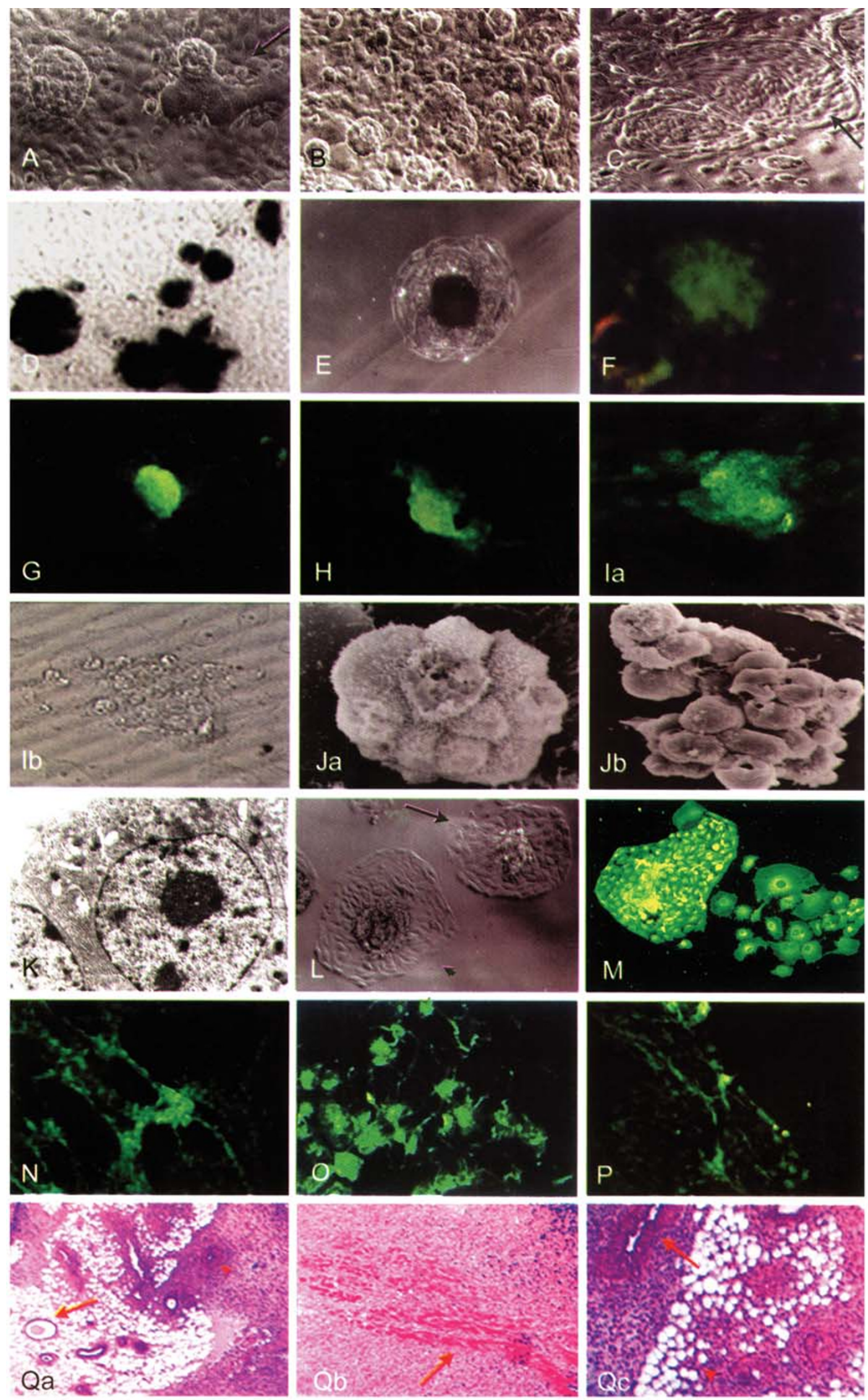


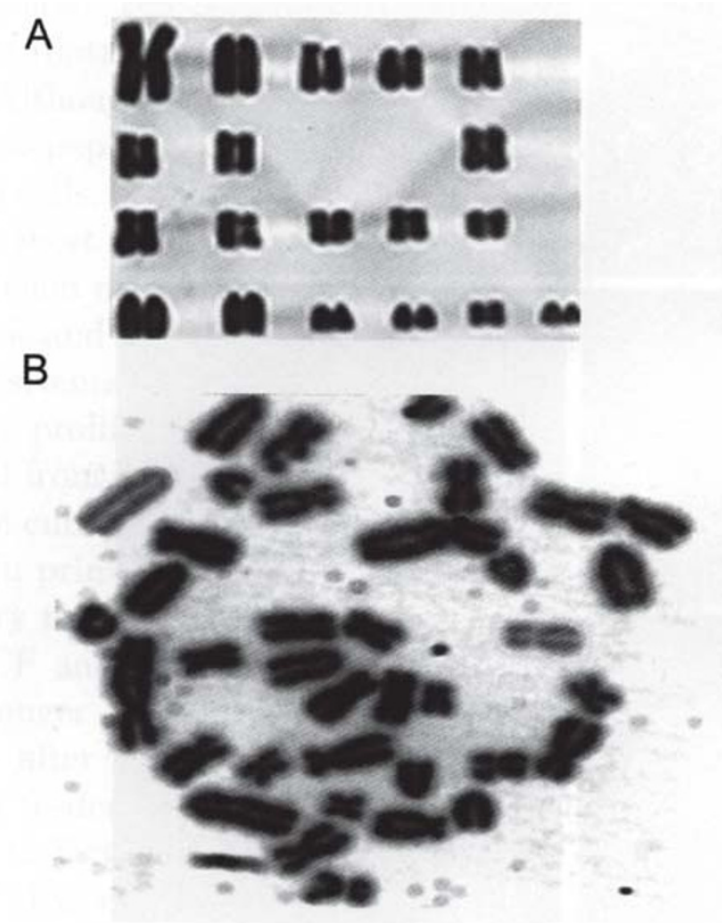

Fig 2. Normal karyotype of BPEG1 cells, 38XX female (A) and its metaphase spread (B).

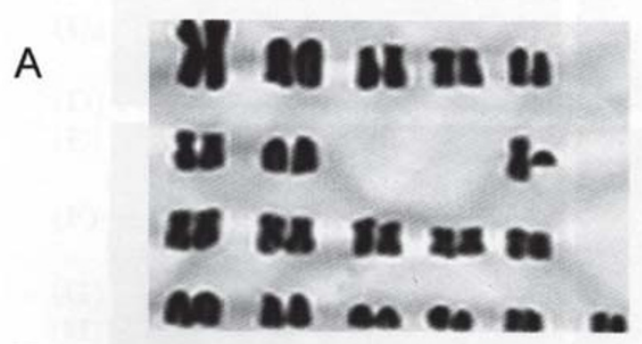

B

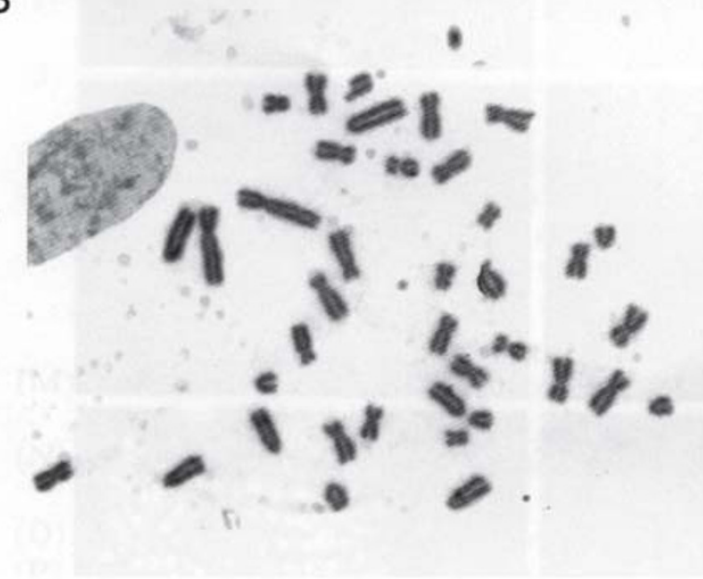

Fig 3. Normal karyotype of BPEG2 cells, 38XY male (A) and its metaphase spread (B).
Mueller et al[13], that SSEA-1 is a good marker for pig PGCs and long term cultured PGCs. However, Takagi et al did not find the expression of SSEA-3 and SSEA-4 in pig PGCs and Mueller et al did not analyze both SSEA-3 and -4. To our knowledge, both markers SSEA-3 and SSEA-4 are present in human EC cells[19], monkey ES cells[20] and human ES and EG cells[21-22]. In our mini-pig EG cells, the simultaneous expression of SSEA-1, SSEA-3 and SSEA-4 antigens seems to suggest that glycosylation in cell surface of EG cells might differ in different species of swine, or more possibly pig PGC and EG cells might be evolutionarily more close to human in the expression pattern of cell surface glycoproteins. Further studies are needed to clarify such discrepancy.

Oct- 4 is a transcription factor that has been considered as a marker for the totipotency of mammalian cells[23]. During mouse development, oct-4 gene is expressed only in pluripotent embryonic cells, such as blastomeres, ICM of blastocysts, epiblasts, primordial germ cells and most of germ cells. Thus, it is also a specific marker for undifferentiated stem cells like ES and EG cells. In this work, pig EG cells display a rather strong positive reaction for Oct-4 staining. Fig 1 Ia, Ib showed the positive immunofluorescent reaction of a pig EG colony with anti-Oct4 antibodies. During differentiation of these cells, the expression of oct-4 gene is rapidly down-regulated and disappeared.

Karyotype analysis was carried out with two EG cells lines after their respective $18^{\text {th }}$ and $20^{\text {th }}$ passages. Mataphasic spreads of two lines were separately analyzed. The number of chromosomes counted in both cell lines showed a normal karyotype, being a female 38XX for BPEG1 cells, and a male 38XY for BPEG2 cells respectively with no visible abnormalities. Fig 2 and Fig 3 represented the metaphasic spreads and karyotypes of these two porcine EG cell lines.

From electron microscopic observations the size of pig EG cells was similar to that of mouse EG cells as seen from scanning microscopic picture at the same magnification, but pig EG cells are obviously more adhered to each other (Fig $1 \mathrm{Ja}, \mathrm{Jb}$ ). Transmission electron microscopic observation demonstrated that cytoplasmic structures of a pig EG cell was rather simple, just like mouse stem cells, con- 
taining spread mitochondria and ribosomes and having a high nuclear to cytoplasmic ratio (Fig $1 \mathrm{~K}$ ).

\section{In vitro and in vivo differentiation}

When the suspension of EG cells was cultured for 4-5 d in hanging drops or onto gelatin-coated wells, simple embryoid bodies (EB) were formed. Outer layer of these EBs were composed of endoderm cells and the core of EBs was undifferentiated EG cells which can still be stained by AKP (see Fig 1E). After those EBs were attached to dish for $5 \mathrm{~d}$, EG cells differentiated into several cell types, usually epithelial-like and fibroblast-like cells (Fig 1L). When EBs derived EG colonies of $12^{\text {th }}$ or $14^{\text {th }}$ passages were induced with $1 \times 10^{-7}-10^{-9} M$ RA for $5 \mathrm{~d}$, then attached to dishes for another 5-10 days, EG cells could give rise to differentiated cell types of three germ layers as evidenced by specific marker staining, such as endoderm cells (Fig 1M), fibroblasts-like cells (Fig $1 \mathrm{~N}$ ), neurons-like cells (Fig 1O), and astrocytes-like cells (Fig 1P).

EG cells formed tumor-like outgrowths after injected subcutaneously into a nude mice for 4-5 w. The outgrowth were examined histologically and found to contain a great variety of cell types, including muscle, glands, neural tube like structure etc and undifferentiated EG-like cells (Fig 1, Qa, Qb and Qc). These results indicated that the EG cells were pluripotent and similar to mouse EG, ES cells in their ability to form tumor-like outgrowths in vivo.

Taken together, the above data can be summarized into the following points: (1) The colony morphology of two porcine EG cell lines is just like that of mouse ES and EG cells. They can maintain such morphology through successive passaging up to over 20 passages. Cryopreseved EG cells from both early and later passages can also keep their growth properties, specific marker and pluripotency in vitro differentiation unchanged after thawing. (2) Biochemical and immunohistochemical features of these EG cells, including the positive AKP staining, the presence of SSEA antigens on their cell surface as well as the expression of totipotent marker protein Oct-4, all point out to their belonging to stem cells. (3) The results of in vitro differentiation of embryoid bodies from these EG cells and the formation of teratoma in nude mice both testified their pluripotent nature. Therefore, save from the confirmation of their ability to form chimera and germline transmission, we have preliminarily established, for the first time, two EG cell lines of Chinese miniswine which can be used for further basic studies.

During the work, the most thing that puzzled us is the low plating efficiency of pig EG cells. We have mentioned it at the beginning of this section, but it is a very complicated problem. The regulation of self renewal of stem cells is a hot topic of research even with mouse ES cells[24]. In our opinion, one way to approach this problem for pig stem cells research is to find out a better feeder cells from a kind of pig somatic cells or even a genetically engineered one, which could provide a much better microenviroment for the survival and proliferation of porcine EG cells than the present-day used mouse STO cells. If that can be realized, then one can develop a much better cell culture system to elevate the plating efficiency of pig EG cells or even ES cells. When we can have a lot of EG cells at hand, many evocative questions can be asked. In this case, we would like to know the function of SSEA antigens and their bearing to the self renewal or differentiation of pig EG cells. But most interestingly, we would like to seek the possibility for the genetic manipulation of pig EG cells or even for the possibility to establish stable pig ES cell lines and their genetic manipulation.

In short, we have successfully established and characterized two EG cell lines from PGCs of Chinese mini-swine. The possible use of these cell lines for further basic studies is discussed.

\section{ACKNOWLEDGEMENTS}

This work was an introductory part of a project supported by National Science Foundation of China (No. 39993430). The authors are very thankful to Dr. JA Piedrahita for his gift of STO cells.

\section{REFERENCES}

1. Yoo D, Giuliri A. Xeno-transplantation and the potential risk of xenogeneic transmission of porcine viruose. Canad J Veteri-research 2000; 64:193-203.

2. Onions D, Cooper DKC, Alexander TJL, et al. An approach to the control of disease transmission in pig-to- human xeno-transplantation. Xeno-transplantation 2000; 7:14355.

3. Bradley A. Production and analysis of chimeric mice. In Teratocarcinomas and embryonic stem cells: A practical Approach. Ed Robertson EJ. Oxford: IRL press:1987: 11352.

4. Campbell KHS, Wilmut I. Totipotency or multipotentiality of culture cells: applications and 
progess. Theriogenology 1997; 47:63-72.

5. Hooper ML. Embryonal stem cells: Introducing planned changes into the animal germline. Chur Swizerland; Harwood Academic publisher, 1992; 1-23.

6. Gerfen RW, Wheeler MB. Isolation of embryonic cell-lines from porcine blastocysts. Anim Biotechnol 1995; 6:1-14.

7. Chen LR, Shiue YL, Bertolni L et al. Establishment of pluripotent cell lines from porcine preimplantation embryos. Theriogenology 1999; 52:195-212.

8. Matsui Y, Zsebo K, Hogan BLM. Derivation of pluripotential embryonic stem cells from murine primordial germ cells in culture. Cell 1992; 70:841-7.

9. Stewart CL, Gali I, Bhatt H. Stem cells from primordial germ cells can reenter the germ lines. Dev Biol 1992; 61: 626-8.

10. Xu X, Yu YS, Tsung HC, Sugano S, Yan YC. The development fate of green fluorescent mouse embryonic germ cells in chimeric embryos. Cell Research 1999; 9:201-8.

11. Shim H, Gutierrez-Adan A, Chen LR, BouDurant RH, Behboodi E, Anderson GB. Isolation of pluripotent stem cells from cultured porcine primodial germ cells. Biol Reprod 1997; 57:1089-95.

12. Piedrahita JA, Moore K, Ortama B, et al. Generation of transgenic porcine chimeras using primordial germ cellderived colonies. Biol Repord 1998; 58:1321-9.

13. Mueller S, Preller K, Rieger N, et al. Chimeric Pigs following blastocyst injection of transgenic porcine primodial germ cells. Mol Reprod Dev 1999; 54:244-54.

14. Lai Liangxue, Koller-Simonds D, Park kwang-wook et al. Production of a-1, 3 galactosyltransferase knockout pigs by nuclear transfer cloning. Science 2002; 295:1089-92.
15. Xu X, Tsung HC, Yan YC. Establishment and differentiation of murine EG cell lines derived from primordial germ cells. Acta Biologiae Exper Sinica 1999; 32:251-63.

16. Solter D, Knowles BB. Monoclonal antibody defining a stage-specific mouse embryonic anigen(SSEA-1). Proc Natl Acad Sci USA 1987; 75:5565-9.

17. Scholer H, S Ruppert, N Suzuki, K Chowdhury, P Gruss. New type of POU domain in germ line-specific protein Oct-4. Nature 1990; 344:435-9.

18. Takagi Y, Talbot NC, Rexroad CE, et al. Identification of pig primordial germ cells by immunocytochemistry and lectin binding. Mol Reprod Dev 1997; 46:567-80.

19. Andrews PW, Oosterhuis JW, Damjanov I. Cell lines from human germ cell tumors. In 揟eratocarcinomas and Embryonic stem cells: A practical Approach? Ed Robertson EJ. Oxford: IRL press, 1987:207-48.

20. Thomson J, Kalishman J, Golos T. Isolation of primates embryonic stem cell line. Proc Natl Acad Sci USA 1995; 92:7844-8.

21. Thomson J, Itskovitz-Eldor J, Shapiro S, et al. Embryonic stem cell lines derived from human blastocysts. Science 1998; 282:1145-7.

22. Shamblott MJ, Axelman J, Wang SP, et al. Derivation of pluripotent stem cells from cultured human primodial germ cells. Proc Natl Acad Sci 1998; 95:13726-31.

23. Pan GJ, Chang ZY, Hans R Schoeler, Pei DQ. Stem cell pluripotency and transcription Factor Oct-4. Cell Research 2002; 161:626-9.

24. Niwa H. Molecular mechanism to maintain stem cell renewal of ES cells. Cell Str and Func 2001; 26:137-48. 